\title{
Gram-positive uropathogens: Empirical treatment and emerging antimicrobial resistance
}

\author{
Umme Laila Urmi $^{1 *}$, Nusrat Jahan ${ }^{1}$, Shamsun Nahar ${ }^{1}$, Masud Rana ${ }^{1}$, Fahmida Sultana ${ }^{1}$, Billal Hossain ${ }^{1}$, Samiul Iqbal ${ }^{2}$, Moyazzem Hossain ${ }^{3}$, \\ Abu Syed Md Mosaddek ${ }^{4}$ and Salequl Islam ${ }^{1}$ \\ ${ }^{1}$ Department of Microbiology, Jahangirnagar University, Savar, Dhaka, Bangladesh \\ ${ }^{2}$ Department of Oral Maxillofacial Surgery, BSMMU, Dhaka, Bangladesh \\ ${ }^{3}$ Department of Statistics, Jahangirnagar University, Savar, Dhaka, Bangladesh \\ ${ }^{4}$ Department of Pharmacology, Uttara Adhunik Medical College, Uttara, Dhaka, Bangladesh
}

\begin{abstract}
Urinary tract infection (UTI) is a global problem. Most UTI research focuses on gram-negative etiology. Enterobacteriaceae was found to be the most prevalent UTI infection constituting more than $80 \%$ of all the reported cases. The major gram-positive bacteria in UTI cases are Staphylococcus saprophyticus, Enterococcus faecalis, Streptococcus agalactiae. Gram-positive pathogens were reported in multiple countries in both uncomplicated and complicated UTI. Antibiotic therapy of gram-positive bacteria is completely different than that of gram-negative UTI pathogens. However, symptoms associated with UTI caused by gram-positive and gram-negative are very similar. Without proper diagnosis, there is a high possibility of getting the wrong diagnosis and subsequent antibiotic therapy. Very limited studies are available focusing etiology and their antibiotic susceptibilities in Bangladesh perspective. We aimed to seek in this gap-filling research area. This study has detected $8.2 \%$ of gram-positive bacteria in UTI patients. The prevalence shows the harmony with the earlier published reports. Male and female were found to be infected equally by gram-positive UTI pathogens. Most of the earlier publication shows that female is more vulnerable to gram-negative UTI bacteria. Unlikely, our findings look males are equally vulnerable by gram-positive in comparison to females. Further studies with more sample sizes can warrant the preliminary findings. Antibiogram analyses showed amikacin and gentamicin as the most effective antibiotics against our tested isolates. In contrast, nitrofurantoin was found the most ineffective drug in this study. Findings of the study could help in prescribing antibiotics from this evidence-based study.
\end{abstract}

Abbreviations: API: Analytic Profile Index; CLSI: Clinical and Laboratory Standards Institute; CLED: Cysteine Lactose Electrolyte Deficient Agar; MDR: Multi Drug Resistant; MHA: Muller Hinton Agar; PBS: Phosphate Buffered Saline; UTI: Urinary Tract Infection

\section{Introduction}

Urinary tract infection (UTI) is one of the most common infectious diseases. The major causative agents of UTIs are Escherichia coli and other Enterobacteriaceae. The remaining proportions are associated with a variety of organisms, including the Gram-positive bacteria Staphylococcus saprophyticus, Enterococcus faecalis, Streptococcus agalactiae (group B Streptococcus, GBS), and other less frequently isolated organisms [1-3]. Most prevalent gram-positive uropathogenic bacteria include Staphylococcus saprophyticus and Enterococcus facecalis [4-6]. Gram-positive uropathogens were reported in multiple countries in both uncomplicated and complicated UTI [7-9]. There are no such exclusive studies have been done in Bangladeshi population to seek gram-positive UTI etiology. Without proper diagnosis, there is a high possibility of getting wrong and inappropriate medications and subsequent adverse side-effects of the associated treatment. Grampositive bacteria are found more often as etiologic agents of UTI among pregnant women and the elderly [10]. Further, complicated UTIs often occur in nosocomial and/or institutional settings and these individuals are at greater risk of gram-positive and polymicrobial UTI [4,5]. It accounts for a large proportion of antibiotic consumption and has a large socioeconomic impact and may contribute to bacterial resistance $[11,12]$. Clinicians often face problems in choosing appropriate antibiotic for empirical therapy for treating UTIs [13]. In Bangladesh, indiscriminate use of antibiotic use brings many adverse outcomes to UTI patients clinically with an extended economic burden. This study aimed to assess the susceptibility profile of gram-positive uropathogens against different prescribe antibiotics. Our study had rationalized the necessity of continued monitoring of urinary tract infected patients for a better understanding of their current epidemiology by gram-positive bacterial strains in the Bangladeshi population.

\section{Methods and materials}

\section{Data collection}

Patient's socio-demographic information, treatment history (whether the patients were treated with an antimicrobial therapy), and any relevant clinical information (kidney stones, pregnancy, recent catheter usage) were recorded by a structured questionnaire.

\section{Study design and specimen collection}

A cross-sectional study was conducted between July 2018 and December 2018 to seek gram-positive bacterial isolate from patients

${ }^{\star}$ Correspondence to: Umme Laila Urmi, Department of Microbiology, Jahangirnagar University, Savar, Dhaka, Bangladesh, Tel: +880-1725996390; E-mail: ummelailaurmi@gmail.com

Key words: uropathogen, UTI, antimicrobial resistance, empirical treatment, Bangladesh

Received: March 22, 2019; Accepted: April 12, 2019; Published: April 16, 2019 
having urinary tract infection. This study was designed based on laboratory investigations of urine samples collected from UTI patients attending in different community hospitals in the Savar area. To actuate the planning, probable UTI patients who sought medical advice at Gonosastha Hospital and Enam Medical College in Savar, Dhaka, were requested to participate in this study. The study includes consented patients having clinical evidence of UTI with symptoms less than seven days presenting at the out-patient department of hospitals and those admitted for less than $48 \mathrm{~h}$.

\section{Bacterial isolation and identification}

To isolate UTI pathogen, clean-catch midstream urine samples were collected in sterile glass tubes and inoculated on a differential culture medium, cysteine-, lactose-, and electrolyte-deficient (CLED) agar (Lyophilchem, Italy). Patients were explained about the standard clean-catch midstream sampling procedure beforehand to avoid contamination with commensal bacteria. To avoid false-positive results, urine specimens were plated within $2 \mathrm{~h}$ after collection. Using CLED agar provides advantages of growth-supports of most potential uropathogens including gram-positive cocci such as Staphylococcus aureus, Staphylococcus saprophyticus, and Enterococcus spp. In parallel, Mannitol Salt Agar (MSA) was inoculated for targeting gram-positive UTI growth. Urine cultures were incubated overnight at $35^{\circ} \mathrm{C}-37^{\circ} \mathrm{C}$ in ambient air. Quantitative urine cultures were done, and colony counts $10^{2}$ or $10^{3} \mathrm{CFU} / \mathrm{mL}$ were considered to define probable UTI infection in the respective patients [14]. Colony counts of less than $10^{2} \mathrm{CFU} / \mathrm{mL}$ were assumed as potential contamination. The purified bacterial colonies were identified by gram staining, conventional biochemical procedures followed by a rapid biochemical-test kit (API 20E, BioMe'rieux, Durham, NC) consisting of a set of chromogenic panels, carbohydrate batteries, and enzymatic substrates. Bacterial identification was validated further by genotyping using $16 \mathrm{~S}$ rRNA of the isolates in part.

\section{Antimicrobial sensitivity assessment}

Isolates were subjected to in vitro antibiotic sensitivity test by standard Kirby-Bauer disc diffusion method and zone of inhibition were interpreted following CLSI (Clinical amp; Laboratory Standards Institute-2010) recommendations. Six antibiotic discs from betalactam, nitrofurantoin, and fluoroquinolone were placed on the bacterial lawn and incubated at $37^{\circ} \mathrm{C}$ to determine the sensitivity pattern. Sensitive bacteria gave a clear zone around the antibiotic disc and zone diameter was measured to compare with the standard.

\section{Detection of $\boldsymbol{\beta}$-lactamase and quinolone-resistant genes}

Isolates that were showing resistant phenotypically were subjected to PCR for the detection of $b l a_{T E M}$ and $q n r S$ gene. A total of six bacterial lysates were prepared. PCR was carried out in PCR tube containing bacterial lysate as DNA template $(1 \mu \mathrm{l})$ with a final volume $12 \mu \mathrm{l}$ containing DNA $2 \mathrm{x}$ premix $(6 \mu \mathrm{l}), 5 \mathrm{pmol}$ of each primer $(1 \mu \mathrm{l})$ and deionized water $(4 \mu \mathrm{l})$. PCR was carried out in a thermal cycler and the cycling condition for $b l a_{T E M}$ and $q n r S$ were: initial denaturation at $94^{\circ} \mathrm{C}$ for $5 \mathrm{~min}$ followed by 32 cycles of amplification with denaturation at $94^{\circ} \mathrm{C}$ for $30 \mathrm{~s}$, annealing at $50^{\circ} \mathrm{C}$ for $30 \mathrm{~s}\left(b l a_{T E M}\right)$ and $55^{\circ} \mathrm{C}$ for $30 \mathrm{~s}$ ( $q n r S$ ) and extension at $72^{\circ} \mathrm{C}$ for $1 \mathrm{~min}$, ending with a final extension at $72^{\circ} \mathrm{C}$ for $7 \mathrm{~min}$. PCR products of the expected size were purified and sequenced using the same primers used to generate the PCR products.

\section{Statistical analyses}

As described, the validated Bangla version of the questionnaire was used for data collection. Completed data collection forms were rechecked by data collection supervisors. Randomly selected $10 \%$ laboratory analyses were done twice by different research person to ensure the authenticity of the laboratory data and to assess interresearcher biases. Verified data were entered and subsequently analyzed using IBM SPSS statistics data editor (version 21). Missing data were excluded from the bivariate analysis. Descriptive statistics were reported as frequency, percentage, and means when applicable along with standard deviation. The antimicrobial susceptibility of test bacteria was evaluated by a 3-type categorical rating scale as susceptible (antibiotic indicated as fully effective), intermediate (indicated as partial effective), and resistant (indicated as non-effective). For the testing association between categorical data, Pearson's chi-square test was used, and Yate's correction for continuity was applied where appropriate. As such the exposure of antimicrobials to the acquisition of antibiotic-resistant gene was calculated. The correlation coefficient was calculated to check the strength of the association. Independent sample t-tests were performed when comparing the means of continuous variables. A twotailed p-value smaller than 0.05 , was considered statistically significant.

\section{Results}

\section{Demographics of the study population}

We approached patients who attended medical doctors of Gonosatha Medical College with their UTI associated complications. We included 147 patients in this study who had severe clinical symptoms. Of them $36(24.5 \%)$ were male and the remaining 111 (75.5\%) were female. Therefore, almost three-fold UTI patients were found female in comparison to their opposite gender (Figure 1). Chisquare test showed female gender associated with a significantly higher number of UTI events $(p=0.04)$. Age distribution of our study cases lies from below one year to above 70 years. However, most frequent UTI infection was in the age group between 21-30 years. Overall agewise case distribution was described in table 1 . A total of five out of 12 Staphylococcus spp (42\%) was identified in the age group 21-30 years. No other trend was observed in age-distribution infection by grampositive UTI infection.

\section{Isolation and identification of gram-positive UTI pathogens}

We examined 147 urine samples from clinically symptomatic patients. CLED culture medium produced different color colonies for different UTI pathogens. Gram-positive Staphylococcus aureus produce yellow colonies and Staphylococcus saprophyticus generates white to yellowish colonies, and Enterococcus spp produces colorless to yellow colonies there. Gram-negative bacteria produce different other colors. Therefore, identifying UTI bacteria by observing colony colors on CLED may be presumptive, but difficult to conclude. We sub-cultured those probable Gram-positive colonies from CLED culture medium on to Mannitol Salt Agar (MSA) and incubated overnight at $37^{\circ} \mathrm{C}$. Growth

Table 1. Age-distribution of UTI study patients

\begin{tabular}{|c|c|c|}
\hline Age in years & Number of UTI patients & Frequency \% \\
\hline $0-0.5$ & 1 & 0.7 \\
\hline $1-10$ & 5 & 3.4 \\
\hline $11-20$ & 23 & 15.6 \\
\hline $21-30$ & 49 & 33.3 \\
\hline $31-40$ & 21 & 14.3 \\
\hline $41-50$ & 27 & 18.4 \\
\hline $51-60$ & 10 & 6.8 \\
\hline $61-70$ & 9 & 6.1 \\
\hline $71-80$ & 2 & 1.4 \\
\hline Total & 147 & 100 \\
\hline
\end{tabular}


colonies on MSA showed white-to-yellowish and pink. Bacteria from MSA were subjected to Gram's staining and found Gram-positive nature. Preliminary gram-positive UTI bacteria have been identified by this study are Staphylococcus species. Analytical profiling index (API) analysis and/or 16sRNA DNA sequencing can be done to identify the etiology into species level. We have detected 12 gram-positive UTI pathogens, which was $8.2 \%$ of the total pathogen population.

\section{Antimicrobial resistance pattern of the UTI gram-positive isolates}

Disc diffusion experiment produced clear zones of bacterial lawn on Muller-Hinton ( $\mathrm{MH}$ ) agar, indicating susceptibility of the respective antibiotics impregnated there. In contrast, resistant isolates did not allow clear zone even in the presence of the antibiotic disc. A mixed trend of the susceptibility against the tested antibiotics was observed in our assessment. The highest resistance was found against nitrofurantoin $(91.7 \%)$, followed by amoxicillin (83\%), ofloxacillin (50\%). Both gentamicin and amikacin were found very effective drugs, where only $8.3 \%$ was found resistant (Figure 2).

\section{Antibiotic resistant genes in gram-positive uropathogens}

We prepared DNA samples from all 12 gram-positive isolates by the heat boiling method. Cell walls of the gram-positive bacteria are usually much more rigid than gram-negative bacteria, which may affect

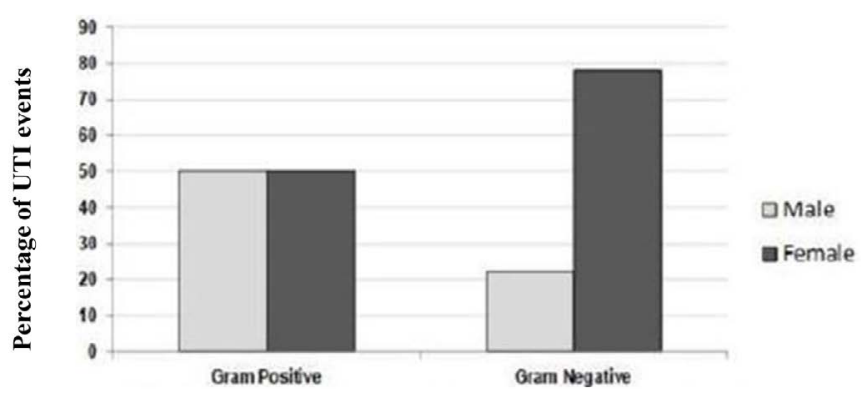

Differential infection by Gram-positive and Gram-negative bacteria

Figure 1. Distribution of gram-positive and gram-negative bacteria among male and female. Female had a significant higher number of UTI than males which was caused by gram-negative bacteria. In the case of gram-positive urinary tract infection, male and female showed equal vulnerability

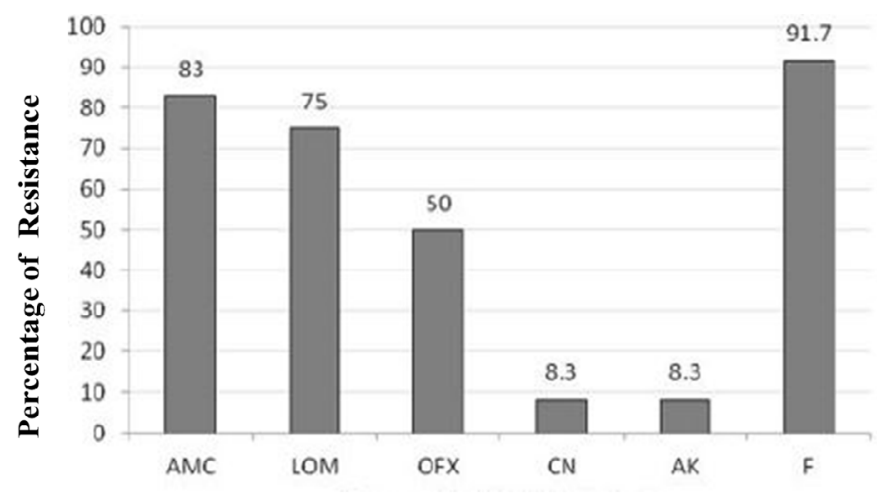

Types of Antibiotics Tested

Figure 2. Prevalence of antibiotic resistance among gram-positive UTI bacteria.

AMC: Amoxicillin; LOM: Lomefloxacin; OFX: Ofloxacin; CN: Gentamicin; AK: Amikacin; F: Nitrofurantoin. Amikacin and Gentamicin appeared most effective antibiotics against Gram-positive UTI bacteria. Nitrofurantoin was found the least effective among the tested antibiotics. liberating DNA in heat-boiling methods. In the current analysis, we examined beta-lactam ring containing antibiotic-resistant gene bla${ }_{T E M}$ and found two isolates $(2 / 6,33 \%)$ isolates positive. Similarly, we checked for fluoroquinolone group antibiotic-resistant gene $q n r-S$ and found three isolates positive $(3 / 6,50 \%)$.

\section{Discussion}

Urinary tract infections represent one of the most common diseases encountered in medical practice, causing significant associated morbidity and occurring from neonates to the elderly $[5,15,16]$. Prevalence of infections may differ with age, sex, and certain predisposing factors. Most of the reported UTI infection was caused by gram-negative bacteria mainly Enterobacteriaceae. There were also strong evidences against gram-positive UTI infection $[8,17]$. We sought to examine the prevalence of gram-positive UTI pathogens in the Bangladeshi population. Empirical treatments for urinary infectionbased severity criteria and clinical symptoms carry great challenge [18]. As antibiotic therapy of gram-positive bacteria is completely different than that of gram-negative UTI pathogens, there is a high possibility of getting the wrong treatment by an empirical approach. Therefore, correct diagnosis with specific antibiotic susceptibility testing is imperative for setting any treatment stewardship. As UTI is a common community-acquired disease in Bangladesh, therefore, a significant percentage of the population are getting infected with this disease each year. The study was designed to check antimicrobial susceptibilities of gram-positive UTI pathogens as well. We detected about $8.2 \%$ gramposition UTI etiologies, notably, Staphylococcus aureus, Staphylococcus saprophyticus, and Enterococcus spp. which attached earlier published reports [19].

Commonly, UTI infection was recorded much higher in females than in males [20]. Unlikely, we found half of the gram-positive infection was reported in males. The preliminary results showed males are more vulnerable by gram-positive in comparison to the overall situation. Age distribution of our study cases lies from below one year to above 70 years. However, most frequent gram-positive UTI infection was in the age group between 21-30 years as supported elsewhere [21].

Antibiotic resistance among uropathogens has become a public health concern in Bangladesh. In this study, antibiotic susceptibility profiles of gram-positive UTI pathogens were determined with six different antibiotic discs a by disc diffusion test. The highest percentage of resistance was observed against nitrofurantoin, whereas the lowest percentage of resistance was found against amikacin and gentamicin. Less than $10 \%$ of the gram-positive uropathognes appeared resistant against those two aminoglycoside group antibiotics. The results support aminoglycoside as a drug of choice for gram-positive UTI pathogens. Among the quinolone group of antibiotics, the highest percentage of resistance had been found by 2 nd generations lomefloxacin (75\%). Our antibiogram findings showed nitrofurantoin was not a very effective drug for gram-positive UTI bacteria. The old beta-lactam antibiotic, amoxicillin was weakly functional against gram-positive UTI pathogens. Fluoroquinolone antibiotics, ofloxacillin and lomefloxacin were found moderate level effective.

The isolates were tested for selected AMR genes by polymerase chain reaction (PCR), using primers specific for the detection of bla${ }_{T E M}$ and $q n r S$. About $33 \%$ isolates were positive for bla- ${ }_{T E M}$ and $50 \%$ was positive for $q n r S$. Antibiotic-resistant genes were detected and showed harmony to the phenotypic susceptibility pattern. Although with utmost sincerity and dedication were investigated to carry out the study it could not go beyond limitations as the sample size was not 
large enough. Despite this limitation hopefully, this finding of the study will help clinicians deciding to prescribe antibiotics from the evidencebased study. The clinician should take care of the specific diagnosis before prescribing empirical antibiotic therapy, particularly for male UTI.

\section{Ethics statement}

This study was approved by the Ethics and Research Review Committee of the Jahangirnagar University, Faculty of Biological Sciences and verbal consent was obtained from UTI patients and doctors.

\section{Acknowledgments}

The authors would like to thank the respective patients and doctors for their cooperation. The authors acknowledge the contribution of staff at the general laboratory, Department of Microbiology, Jahangirnagar University.

\section{Disclosure}

This work was partly supported by the Grant for Advance Research (GARE), Ministry of Education and the Ministry of Science and Technology Research Grant, Bangladesh, to Dr. Md. Salequl Islam.

\section{References}

1. Stamm WE, Norrby SR (2001) Urinary tract infections: disease panorama and challenges. J Infect Dis 183: S1-S4. [Crossref]

2. Hooton TM (2012) Clinical practice. Uncomplicated urinary tract infection. $J$ Infect Dis 366: 1028-1037. Epub 2012/03/16. [Crossref]

3. Piette A, Verschraegen G (2009) Role of coagulase-negative staphylococci in human disease. Vet Microbiol 134: 45-54. [Crossref]

4. Edwards MS, Baker C (2005) Group B streptococcal infections. GROUP 12:c0060. [Crossref]

5. Matthews SJ, Lancaster JW (2011) Urinary tract infections in the elderly population. Am J Geriatr Pharmacother 9: 286-309. [Crossref]

6. Kline KA, Lewis AL (2016) Gram-positive uropathogens, polymicrobial urinary tract infection, and the emerging microbiota of the urinary tract. Microbiol Spectr 4: 2. [Crossref]
7. Clouse K, Shehabi A, Suleimat AM, Faouri S, Khuri-Bulos N, et al. (2019) High prevalence of Group B Streptococcus colonization among pregnant women in Amman, Jordan. BMC pregnancy and childbirth 19: 177.

8. de Sousa VS, da-Silva APS, Sorenson L, Paschoal RP, Rabello RF, et al. (2017) Staphylococcus saprophyticus Recovered from Humans, Food, and Recreational Waters in Rio de Janeiro, Brazil. Int J Microbiol 2017: 4287547 [Crossref]

9. Lee DH, Klinkova O, Kim JW, Nanjappa S, Greene JN (2019) A Case Series of Staphylococcus lugdunensis Infection in Cancer Patients at an Academic Cancer Institute in the United States. Infect Chemo 51: 45-53. [Crossref]

10. Kunin CM (1994) Urinary tract infections in females. Clin Infect Dis 18: 1-10. [Crossref]

11. Croxen MA, Finlay BB (2010) Molecular mechanisms of Escherichia coli pathogenicity. Nat Rev Microbiol 8: 26-38. [Crossref]

12. Grude N, Tveten Y, Kristiansen BE (2001) Urinary tract infections in Norway: bacterial etiology and susceptibility. A retrospective study of clinical isolates. Clin Microbiol Infect 7: 543-547. [Crossref]

13. Falagas ME, Giannopoulou KP, Kokolakis GN, Rafailidis PI (2008) Fosfomycin: use beyond urinary tract and gastrointestinal infections. Clin Infect Dis 46: 1069-1077. [Crossref]

14. Stamm WE, Counts GW, Running KR, Fihn S, Turck M, et al. (1982) Diagnosis of coliform infection in acutely dysuric women. $N$ Engl J Med 307: 463-468. [Crossref]

15. Detweiler K, Mayers D, Fletcher SG (2015) Bacteruria and Urinary Tract Infections in the Elderly. Urol Clin North Am 42: 561-568. [Crossref]

16. Arshad M, Seed PC (2015) Urinary tract infections in the infant. Clin Perinatol 42 17-28. [Crossref]

17. Tien BYQ, Goh HMS, Chong KKL, Bhaduri-Tagore S, Holec S, et al. (2017) Enterococcus faecalis Promotes Innate Immune Suppression and Polymicrobial Catheter-Associated Urinary Tract Infection. Infect Immun pii: e00378-17. [Crossref]

18. Vasquez V, Ampuero D, Padilla B (2017) Urinary tract infections in inpatients: that challenge. Rev Esp Quimioter 30: S39-S41. [Crossref]

19. Hooton TM, Stamm WE (1997) Diagnosis and treatment of uncomplicated urinary tract infection. Infect Dis Clin North Am 11: 551-581. [Crossref]

20. Medina M, Castillo-Pino E (2019) An introduction to the epidemiology and burden of urinary tract infections. Ther Adv Urol 11: 1756287219832172. [Crossref]

21. Wallmark G, Arremark I, Telander B (1978) Staphylococcus saprophyticus: a frequent cause of acute urinary tract infection among female outpatients. J Infect Dis 138: 791797. [Crossref]

Copyright: C2019 Urmi UL. This is an open-access article distributed under the terms of the Creative Commons Attribution License, which permits unrestricted use, distribution, and reproduction in any medium, provided the original author and source are credited. 